\title{
A Prospective Randomized Controlled Trial: Short- Time In Vitro Maturation For Improvement of Oocytes Quality And Reproductive Outcomes
}

Ying-ying Liu

Sun Yat-Sen University https://orcid.org/0000-0001-9595-7208

Ya-nan Shi

Sun Yat-Sen University

Hai-tao Zeng

Sun Yat-Sen University

Cong Fang

Sun Yat-Sen University

Ri-cheng Chian

Tenth Hospital of Tongji University

Xiao-yan Liang ( $\sim$ liangxy2@mail.sysu.edu.cn )

Sun Yat-Sen University

\section{Research Article}

Keywords: short-term IVM medium, In vitro fertilization, Oocyte maturity, Aavailable embryos

Posted Date: February 1st, 2022

DOl: https://doi.org/10.21203/rs.3.rs-796785/v1

License: (9) (i) This work is licensed under a Creative Commons Attribution 4.0 International License.

Read Full License 


\section{Abstract}

Background: Current standard stimulation protocols use gonadotropins (recombinant or hMG) combined with LHRH agonists to prevent premature ovulation to obtain an average of 10-15 mature oocytes per retrieval. However, it usually has some immature oocytes retrieved with mature oocytes even after triggering LH surge. Compared to fertilization medium, IVM medium may be more effective in stimulating the maturation of oocyte nucleoplasm. This study aimed to explore the effect of short-term culture in our novel IVM medium on oocyte maturity and subsequent embryonic development in current standard stimulation protocols.

Methods/Design: This study is a multicenter, prospective, randomized controlled clinical trial (1:1 treatment ratio of short term IVM group or control group). we will need to enroll 1860 participants in each group; that is, a total of 3720 participants. The The retrieved cumulus-oocyte complexes $₫ \operatorname{COCs} \rrbracket$ of the short-term IVM group were exposed to IVM medium immediately after retrieval and subsequently incubated for 4 hours, and COCs in control group were cultured in G-IVF PLUS in 4-wells culture dish after retrieval. The primary study endpoint is number of available embryos: defined as number of embryos $d \geq 4$ cells and $\leq 30 \%$ fragmentation (except embryos developed from $\geq 3$ PN zygotes) on day 3 of observation. All tests were two-tailed, and differences with $p$ value $<0.001$ for interim analysis or $p$-value $<0.05$ for final analysis are considered statistically significant.

Discussion:This study aimed to explore the effect of short-term culture in our novel IVM medium on oocyte maturity and subsequent embryonic development.

Trial registration: chictr.org.cn Identifier:ChiCTR2000038043

\section{Background}

The development of follicles presents multiple batches of development, including eggs of different developmental and mature stages obtained in egg retrieval during the treatment of ovulation induction [1]. Current standard stimulation protocols use gonadotropins (recombinant or hMG) combined with LHRH agonists to prevent premature ovulation to obtain an average of 10-15 mature oocytes per retrieval[2]. However, it usually has some immature oocytes retrieved with mature oocytes even after triggering LH surge [3]. Practically, those immature oocytes retrieved were discarded. In addition, insemination is performed normally after approximately $41 \mathrm{~h}$ of HCG injection. Therefore, there are a few hours (2-5 h) after oocyte retrieval before insemination in fertilization medium (FM) [4-5]. We do not know whether or not FM is an optimal medium for oocyte maturation.

It is found that the composition of FM and follicles fluid (FF) is quite different, with FM lacking of factors that promote the maturation of oocytes, and may lead to low development potential[6]. Traditional in vitro maturation (IVM) is the in vitro culture of immature oocytes after their recovery from follicles not previously exposed to either exogenous LH or hCG prior to retrieval[7]. During IVM, such immature oocytes progress from prophase I stage through meiosis I reached metaphase II(MII) [8]. Cumulus 
granulosa cells play a key role in the maturation of eggs and the inhibition of aging after maturation. During the initiation and egg maturation, gonadotropins and growth factors regulate egg maturation by acting on cumulus granulosa cells. FSH/LH stimulated IP3/Ca2+ pathway by binding to receptors on cumulus granulosa cells and reduce the level of cAMP and purine substances, promoting the level of oocyte $\mathrm{Ca} 2+$ concentration and inducing the secretion of many nuclear maturation factors[9]. A large number of studies have shown that FSH cannot promote oocyte maturation, but promoted the expression of $\mathrm{LH}$ receptors[10]. Although adding FSH to IVM medium cannot directly improve oocyte maturation rate, it can significantly increase oocyte fertilization rate and blastula formation rate[10-13].

Besides the hormone, oocyte-derived factors like growth differentiation factor 9 (GDF-9) can also regulate the function of oocytes and cumulus granulosa cells through paracrine and autocrine mechanisms. Previous studies have found that GDF-9 concentrations in FF collected during the ovarian stimulation cycle are significantly associated with oocyte nuclear maturation and embryo quality [14-15].

Above all, conventional IVM medium contains FSH, LH, GDF-9, and other factors for promoting oocyte nuclear maturation[16]. Compared to FM, IVM medium may be more effective in stimulating the maturation of oocyte nucleoplasm.

To improve the oocyte maturation rate and embryo quality, oocytes were cultured in IVM medium for short-term instead of FF after retrieval before insemination is performed. This short-term exposure to IVM medium may be beneficial for women with a lower yield of mature oocytes after ovarian stimulation. Our previous research indicated that short-term IVM using Sages IVM medium could increase the rate of highquality embryos. In this study, oocytes were cultured for short-term in a novel IVM medium, supplemented with forskolin, FSH, GDF-9 and EGF, etc., which could promote the late maturation and delay the in vitro aging of oocytes, so that promoting the synchronization of nucleocytoplasmic maturation. In brief, this study aimed to explore the effect of short-term culture in our novel IVM medium on oocyte maturity and subsequent embryonic development.

\section{Methods/design}

\section{Inclusion criteria}

1. Women who have a $\geq 1$-year history of infertility.

2. Women with $\geq 20$ and $<45$ years old, $A M H>1.1 \mathrm{ng} / \mathrm{ml}$, BMI between $18.5 \mathrm{~kg} / \mathrm{m}^{2}$ and $24.5 \mathrm{~kg} / \mathrm{m}^{2}$.

3. Women who have at least one of the following indications for IVF or ICSI:

a. Ovulation dysfunction and failed to get pregnant after ovulation induction treatment.

b. Tubal factors: unilateral or bilateral tubal obstruction, adhesion, unilateral or bilateral salpingectomy or tubal ligation. 
4. Women who are undergoing their first or second cycle of IVF or ICSI.

5. Women whose retrieved oocytes number is more than six.

6. Fresh semen is collected on Ovum-pick-up day.

7. One or two embryos with top quality will be transferred on day 3 of a fresh cycle, and one blastocyst with top quality will be transferred on day 5 of the cycle.

\section{Exclusion criteria}

1. Couple with a contraindication for ICSI, including poorly controlled type 1 or type 2 diabetes mellitus; undiagnosed liver disease or dysfunction (based on serum liver enzyme test results); renal disease or abnormal serum renal function; anemia; history of deep venous thrombosis, pulmonary embolus or cerebrovascular accident; uncontrolled hypertension or known symptomatic heart disease; history of (or suspected) cervical carcinoma, endometrial carcinoma or breast carcinoma and unexplained colporrhagia.

2. Couples receiving donor sperm or donor eggs.

3. Couples undergoing preimplantation genetic testing.

4. Women with less than 6 oocytes retrieved.

6. Using frozen semen.

\section{Interventions}

\section{Randomization}

Randomization and allocation of eligible patients to study groups were performed on the day before oocyte retrieval. This procedure was performed by administrative staff in the trial center not involved in the treatment procedure, using an online trial system with a computer-generated randomization list that allocates couples in a 1:1 ratio to short term IVM group or control group.

\section{Controlled ovarian hyperstimulation and oocyte retrieval}

Patients undergoing their first IVF cycle where ganirelix acetate (Ganirelix; Organon Pharmaceuticals, West Orange, NJ) was used for pituitary suppression in a standard $\mathrm{GnRH}$ antagonist protocol. For the above treatments, menstrual cycle of patient includes spontaneous menstrual cycle, and irregular menstrual cycle by using oral contraceptives or progestins. Before gonadotrophin treatment, baseline pelvic ultrasound, as well as basic serum hormones (such as $\mathrm{FSH}$, luteinizing hormone, progesterone (P) and $\beta$-human chorionic gonadotropin ( $\beta$-hCG)) was measured to confirm the follicle status. The initial dosage of gonadotrophin (Gonal-F) is $150-300 \mathrm{mg}$ /day and the subsequent dose was adjusted according to the individual response. Gonadotrophin treatment was continued till the trigger day. After 
two or more follicles reached a diameter $\geq 18 \mathrm{~mm}, 250 \mu \mathrm{g}$ of hCG (Ovitrelle, $250 \mu \mathrm{g} \mathrm{sc}$ ) was injected on trigger day.

Oocyte retrieval is scheduled for 36 hours $( \pm 2)$ after hCG injection. Routine oocyte pick-up was performed under transvaginal ultrasound guidance via 17-18G oocyte aspiration needle using intravenous sedation.

\section{In vitro fertilization and embryo culture}

\section{Oocyte retrieval and preparation}

Before egg retrieval, the IVM medium and FF were equilibrated overnight in a humidified $37^{\circ} \mathrm{C}$ of $5 \% \mathrm{CO} 2$ incubator. The IVM medium was supplemented with forskolin, FSH囚GDF-9 and EGF. The retrieved cumulus-oocyte complexes ( $\mathrm{COCs}$ ) were placed in different culture medium covered by lightweight paraffin oil and incubated in a humidified $37^{\circ} \mathrm{C}, 5 \% / 6 \% \mathrm{CO} 2$ incubator after oocyte retrieval immediately for 2-6 hours before injection. The intact COCs of the control group were cultured in G-IVF PLUS (Vitrolife Sweden AB, Goteborg, Sweden) in 4-wells culture dish after retrieval. The COCs of the short-term IVM group were exposed to IVM medium immediately after retrieval and subsequently incubated for 4 hours before ICSI. Following this short-term IVM exposure, gametes handling was identical to the untreated group.

\section{Semen preparation}

Fresh ejaculated semen samples were obtained by masturbation after 2-7 days' abstention from sexual intercourse on the day of oocyte retrieval. Sperm concentration and progressive motility are assessed according to the fifth edition of WHO laboratory standards of human semen. Semen samples were prepared by discontinue density gradient centrifugation or swim-up protocol according to local routines [17].

Prior to ICSI, COCs were exposed to $80 \mathrm{IU} / \mathrm{mL}$ hyaluronidase (SAGE, Cooper Surgical Inc., USA) for 30 to $45 \mathrm{~s}$ and then mechanically denuded using $155-\mu \mathrm{m}$ stripper pipettes (Research Instruments Ltd, UK). The denuded oocytes were examined to assess integrity and maturity. Only those oocytes that have extruded the first polar body (metaphase-Il oocytes) were microinjected after 4 hours of IVM (short-term IVM group) or G-IVF PLUS (control group) culture.

\section{Assessment of fertilization and embryo quality}

Apart from the IVM culture, assisted reproductive treatments were similar for the two groups. Whether inseminated or injected, oocytes were assessed for pronuclei status at the fertilization check 16-18 $\mathrm{h}$ later. Zygotes with 2PN were incubated in $25 \mu \mathrm{L}$ drops of G1 (Vitrolife Sweden AB, Goteborg, Sweden) and overlain with $8 \mathrm{~mL}$ equilibrated mineral oil (Vitrolife Sweden AB, Goteborg, Sweden) in Falcon 3001 culture dishes (Becton Dickinson Labware, Franklin Lakes, NJ, USA). Gamete and embryo cultures were maintained at $37^{\circ} \mathrm{C}$ in a humidified atmosphere of $5 \% \mathrm{CO} 2$ atmosphere. On day 3, embryos were graded 
for cell number, fragmentation (scores 0 through 4), and symmetry (scores 1 through 3 ) using standard criteria as previously described [18]. Good quality embryos were defined as those with a cell number greater or equal to 8 , fragmentation score of 0 or 1 , and symmetry score of 1 or 2 .

\section{Embryo transfer and luteal phase support}

Transfer of fresh embryos is the usual practice when fresh embryos are available in our study centers. In some cases, all embryos may be cryopreserved without a fresh-embryo transfer, most commonly to prevent ovarian hyperstimulation syndrome (OHSS). In this condition, patients was dropped from our program. Physicians performed fresh embryo transfer in 3 following the day of oocytes collection for participants to receive fresh embryo transfer. Luteal support, Participants in both groups received luteal phase support for 2 weeks with vaginal progesterone obtained on the oocyte retrieval day. If the patient is pregnant, luteal phase support continued till 10 weeks gestation.

All patients of thawed cycle underwent endometrial preparation with HRT. Patients with uterine diseases (e.g., fibroids, polyps, and previously diagnosed Müllerian abnormalities), the presence of hydrosalpinx or an endometrial thickness $<7 \mathrm{~mm}$ after HRT were excluded.

\section{Pregnancy evaluation}

Serum $\beta$-hCG was measured to determine pregnancy 14 days after embryo transfer. If a biochemical pregnancy has been achieved, a transvaginal ultrasound scan was obtained 35 days after embryo transfer to evaluate the clinical pregnancy. The ultrasound scan was repeated at 11 weeks gestation to confirm ongoing pregnancy if a biochemical pregnancy has been achieved.

\section{Follow-up evaluation}

At 12 weeks gestation, the presence of first-trimester pregnancy complications (OHSS, miscarriage, ectopic pregnancy and/or gestational trophoblastic disease) was evaluated by inspecting medical records and recorded by completing the case report form for the first pregnancy follow-up time point.

\section{Outcome measurements}

\section{Primary outcome}

The primary study endpoint is number of available embryos: defined as number of embryos $d \geq 4$ cells and $\leq 30 \%$ fragmentation (except embryos developed from $\geq 3$ PN zygotes) on day 3 of observation.

\section{Secondary outcomes}

1. The number of high-quality embryos on the third day.

2. Maturation rate.

3. Fertilization: defined as the number of zygotes with 2PN (per woman randomized and per oocyte retrieved). 
4. Total fertilization failure: defined as no oocyte formed 2PN in this given cycle.

5. The number of blastocyst formation.

6. Implantation rate: defined as the number of gestational sacs observed per embryo transferred.

7. Clinical pregnancy: defined as one or more observed gestational sac or definitive clinical signs of pregnancy under ultrasonography at the 7 th week of gestation (including clinically documented ectopic pregnancy).

8. Cumulative pregnancy rate: calculated by dividing the sum of fresh and frozen pregnancies by the number of cycles with transfer.

9. Multiple pregnancies: defined as a pregnancy with two or more gestational sacs or positive heartbeats at the 7th week of gestation.

10. Ongoing pregnancy: defined as the presence of a gestational sac and fetal heartbeat after 12th week of gestation.

\section{Statistical analysis}

For continues variables, parameters normally distributed were expressed as mean with SD and compared using Student's t-test. If the parameters are non-normally distributed, their medians and IQRs were reported. Furthermore, the Mann-Whitney $\mathrm{U}$ test was utilized to test the distribution of these variables. For categorical variables, we present the proportion between each group and distributions were compared using Pearson's $\chi 2$ test and Fisher's exact test when appropriate. Data analysis of this trial followed the intention-to-treat principle, in which all randomized women were considered in the primary comparison between treatment groups. Per-protocol analysis was conducted as a secondary analysis in participants who complied with protocol. For missing values, a range of clinically plausible scenarios was used to impute missing values to test the robustness of the findings. For losses to follow-up and protocol violations, we used sensitive analyses to explore the effect of these factors on the trial findings. All tests were two-tailed, and differences with $p$ value $<0.001$ for interim analysis or $p$-value $<0.05$ for final analysis are considered statistically significant. All statistical analyses were performed with the SAS software package. The statistical analysis was performed by an independent statistician. The analysis is described in detail in a statistical analysis plan.

\section{Sample size and power calculations}

Among recruited women囚ages $\geq 20$ and $<40$ years old, $A M H>1.1 \mathrm{ng} / \mathrm{ml}$, the average live birth rate after IVF/ICSI during 2014-2018 calculated overall study sites was 50\% per cycle. Based on previous studies about fertility care as well as the discussion by gynecologists and methodologists, we assumed that the minimal clinically important difference to make short-term IVM medium preferable over conventional medium would be $5 \%[19]$. To demonstrate this difference with a two-sided test, $5 \%$ alpha-error, $90 \%$ statistical power and taking into consideration a dropout of 10\%, we will need to enroll 1860 participants in each group; that is, a total of 3720 participants (the ratio between groups will be 1:1). For the interim 
analysis, we used the Haybittle-Peto boundary. The significance level for the interim analysis is 0.001 and for the final analysis $0.05[20]$.

\section{Trial status}

The study was conceived and designed in 2021. The first participant will be randomized in January 2022. We plan to complete recruitment in January 2024.

\section{Abbreviations}

$\mathrm{COH}$ : Controlled ovarian hyperstimulation; E2: Estradiol; FSH: Folliclestimulating hormone; GnRH: Gonadotropin-releasing hormone; hCG: Human chorionic gonadotropin; ICSI: Intracytoplasmic sperm injection; IVF: In vitro fertilization; OHSS: Ovarian hyperstimulation syndrome; SGA: Small for gestational age

\section{Declarations}

\section{Competing interests}

The authors declare that they have no competing interests.

\section{Authors' contributions}

XL designed the whole study. YL and YS supervised the whole project and performed

data analysis. YL supervised patient diagnosis and recruitment. $Y L$ and $Y S$ conducted data analyses and drafted the manuscript.YS,HZ,CF and RQ participated in the manuscript writing and laboratory procedure design. All authors critically reviewed the article and approved the final manuscript.

\section{Funding}

National Key R\&D Program of China (2017YFC1001604); National Natural Science Foundation of China (81801449).

\section{Author details}

${ }^{*}$ Correspondence address $₫ 3^{\text {rd }}$ floor, Buididng B, Reproductive Medical Center of the Sixth Affiliated Hospital,Sun Yat-Sen University,No.17 Shougouling Road,Tianhe district,Guangzhou,510655,China. Email: Liangxy2@mail.sysu.edu.cn

${ }^{1}$ Yingying Liu and Yanan Shi contributed equeally to this work.

${ }^{1-3}$ Reproductive Medical Center of the Sixth Affiliated Hospital,Sun Yat-Sen University,No.17 Shougouling Road,Tianhe district,Guangzhou,510655,China 
${ }^{4}$ Reproductive center,Tenth Hospital of Tongji University

\section{References}

1. Mao L, Lou H, Lou Y, et al. Behaviour of cytoplasmic organelles and cytoskeleton during oocyte maturation. Reproductive Biomedicine Online. 2014;28(3):284-99.

2. Ovarian Stimulation The Eshre Guideline Group On,Bosch Ernesto,Broer. Simone, et al. ESHRE guideline: ovarian stimulation for IVF/ICSI.Hum Reprod Open, 2020, 2020: hoaa009.

3. Kim B, Lee S, Kim K, Han C, Kim J. In vitro maturation, fertilization, and development of human germinal vesicle oocytes collected from stimulated cycles. Fertil Steril. 2000;74(6):1153-8.

4. Trounson A, Anderiesz C, Jones G. Maturation of human oocytes in vitro and their developmental competence. Reproduction. 2001;121:51-75.

5. Gilchrist RB, Luciano AM, Richani D, Zeng HT, Wang X, Vos MD,et al. Oocyte maturation quality: role of cyclic nucleotidesReproduction. 2016;152(5):R143-57.

6. Reichman D, Politch J, Ginsburg E, Racowsky C. Extended in vitro maturation of immature oocytes from stimulated cycles: an analysis of fertilization potential, embryo development, and reproductive outcomes. J Assist Reprod Genet. 2010;27:347-56.

7. Chian RC, Nargund G, Huang JYJ, editors, Development of In Vitro Maturation for Human Oocytes: Natural and Mild Approaches to Clinical Infertility Treatment. Springer Press, 1st Edition, 2017.

8. Wang N, Le F, Zhan QT, et al. Effects of In Vitro Maturation on Histone Acetylation in Metaphase II Oocytes and Early Cleavage Embryos. Obstet Gynecol Int. 2010;2010(2):989278.

9. Gilchrist RB, Luciano AM, Richani D, Zeng HT, Wang X, Vos MD, Sugimura S, Smitz J, Richard FJ, Thompson JG. Oocyte maturation and quality: role of cyclic nucleotides. Reproduction. 2016;152(5):R143-57.

10. Sirard MA, Desrosier S, Assidi M.. In vivo and in vitro effects of FSH on oocyte maturation and developmental competence. Theriogenology, 2007, 68.

11. Walls M, Hart R, Keelan J, Ryan J. Structural and morphologic differences in human oocytes after in vitro maturation compared with standard in vitro fertilization. Fertil Steril. 2016;106(6):1392-8. e5.

12. Appeltant R, Somfai T, Maes D, A VANS, Kikuchi K. Porcine oocyte maturation in vitro: role of cAMP and oocyte-secreted factors - A practical approach. J Reprod Dev. 2016;62(5):439-49.

13. Liu X, Xie F, Zamah AM, Cao B, Conti M. Multiple pathways mediate luteinizing hormone regulation of cGMP signaling in the mouse ovarian follicle. Biol Reprod. 2014;91(1):9.

14. Yeo CX, Gilchrist RB, Thompson JG, et al. Exogenous growth differentiation factor 9 in oocyte maturation media enhances subsequent embryo development and fetal viability in mice. Hum Reprod. 2007;23(1):67-73.

15. Hussein TS, Thompson JG, Gilchrist RB. Oocyte-secreted factors enhance oocyte developmental competence. Dev Biol. 2006;296(2):514-21. 
16. Zeng HT, Richani D, Sutton-McDowall ML, et al. Prematuration with cyclic adenosine monophosphate modulators alters cumulus cell and oocyte metabolism and enhances developmental competence of in vitro-matured mouse oocytes. Biol Reprod. 2014 Aug;91(2):47.

17. Cong Fang J, Tang R, Huang, et al. Comparison of IVF outcomes using conventional insemination and ICSI in ovarian cycles in which only one or two oocytes are obtained. J Gynecol Obstet Biol Reprod. 2012 Jul 20.

18. Cong Fang R, Huang L-NW, Jia L. Frozen-thawed day 5 blastocyst transfer is associated with a lower risk of ectopic pregnancy than day 3 transfer and fresh transfer. Fertil Steril. 2015 Mar;103(3):6551.e3.

19. Yang ZY, Chian RC. Development of in vitro maturation techniques for clinical applications. Fertil Steril. 2017;108(4):577-84.

20. Blenkinsop A, Parmar MK, Choodari-Oskooei B. Assessing theimpact of effcacy stopping rules on the error rates under the multiarm multi-stage framework. Clin Trials 2019;1496049025.

\section{Figures}


STUDY PERIOD

\begin{tabular}{|c|c|c|c|c|c|c|c|c|c|c|}
\hline TimePoint & ENROLYENT & $\begin{array}{l}\text { OVULATION } \\
\text { PROTOCOL }\end{array}$ & $\begin{array}{l}\text { OOCYTE } \\
\text { PICK-UP } \\
\text { DAY }\end{array}$ & $\begin{array}{l}\text { 1DAY } \\
\text { AFTER } \\
\text { OOCYTE } \\
\text { PICK-UP }\end{array}$ & $\begin{array}{l}3 \text { DAY } \\
\text { AFTER } \\
\text { OOCYTE } \\
\text { PICK-UP }\end{array}$ & $\begin{array}{l}\text { 5DAY } \\
\text { AFTER } \\
\text { OOCYTE } \\
\text { PICK-UP }\end{array}$ & $\begin{array}{l}14 \text { DAYS } \\
\text { AFTER } \\
\text { EXBRYO } \\
\text { TRANSPL } \\
\text { ANTATIO } \\
\text { N }\end{array}$ & $\begin{array}{l}30 \text { DAYS } \\
\text { AFTER } \\
\text { ENBRYO } \\
\text { TRANSPL } \\
\text { ANTATIO } \\
\text { N }\end{array}$ & $\begin{array}{l}12 \\
\text { TEEKS } \\
\text { PREGNAN } \\
\text { CY }\end{array}$ & $\begin{array}{l}\text { PREGN } \\
\text { ANCY } \\
\text { OUTCO } \\
\text { ME }\end{array}$ \\
\hline $\begin{array}{l}\text { Informed } \\
\text { consent }\end{array}$ & $\sqrt{ }$ & & & & & & & & & \\
\hline $\begin{array}{l}\text { Enrolled/ } \\
\text { exclusive } \\
\text { criterion }\end{array}$ & $\sqrt{ }$ & & & & & & & & & \\
\hline $\begin{array}{l}\text { Randomizatio } \\
\mathrm{n}\end{array}$ & & & $\sqrt{ }$ & & & & & & & \\
\hline $\begin{array}{l}\text { Medical } \\
\text { record }\end{array}$ & & & & & & & & & & \\
\hline $\begin{array}{l}\text { Ultrasonic } \\
\text { record }\end{array}$ & $\sqrt{ }$ & $\sqrt{ }$ & & & & & & & & \\
\hline $\begin{array}{l}\text { Oocyte } \\
\text { retrieval } \\
\text { record }\end{array}$ & & & $\sqrt{ }$ & & & & & & & \\
\hline $\begin{array}{l}\text { Embryo } \\
\text { quality } \\
\text { evaluation }\end{array}$ & & & & $\sqrt{ }$ & $\sqrt{ }$ & & & & & \\
\hline $\begin{array}{l}\text { Pregnency } \\
\text { outcome }\end{array}$ & & & & & & & & & & $\sqrt{ }$ \\
\hline $\begin{array}{l}\text { Medication } \\
\text { administrati }\end{array}$ & & & & & $\sqrt{ }$ & $\sqrt{ }$ & $\sqrt{ }$ & $\sqrt{ }$ & $\sqrt{ }$ & $\sqrt{ }$ \\
\hline \multicolumn{11}{|l|}{ on record } \\
\hline $\begin{array}{l}\text { Adverse } \\
\text { event }\end{array}$ & & $\sqrt{ }$ & $\sqrt{ }$ & $\sqrt{ }$ & & $\sqrt{ }$ & $\sqrt{ }$ & $\sqrt{ }$ & $\sqrt{ }$ & $\sqrt{ }$ \\
\hline
\end{tabular}

Figure 1

STUDY PERIOD

\section{Supplementary Files}

This is a list of supplementary files associated with this preprint. Click to download. 
- completedSPIRITchecklist.docx 